\title{
Optimal Short Driving Mission Control for a Diesel-Electric Powertrain
}

\author{
Martin Sivertsson and Martin Eriksson
}

\section{Linköping University Post Print}

N.B.: When citing this work, cite the original article.

(C2012 IEEE. Personal use of this material is permitted. However, permission to reprint/republish this material for advertising or promotional purposes or for creating new collective works for resale or redistribution to servers or lists, or to reuse any copyrighted component of this work in other works must be obtained from the IEEE.

Martin Sivertsson and Martin Eriksson, Optimal Short Driving Mission Control for a DieselElectric Powertrain, 2012, IEEE VPPC 2012 -- The 8th IEEE Vehicle Power and Propulsion Conference.

Postprint available at: Linköping University Electronic Press

http://urn.kb.se/resolve?urn=urn:nbn:se:liu:diva-84956 


\title{
Optimal Short Driving Mission Control for a Diesel-Electric Powertrain
}

\author{
Martin Sivertsson and Lars Eriksson \\ Vehicular Systems, Dept. of Electrical Engineering \\ Linköping University, SE-581 83 Linköping, Sweden \\ Email:\{marsi,larer\}@isy.liu.se
}

\begin{abstract}
Time and fuel optimal control for a diesel-electric powertrain in transient operation is studied using a four state, three controls non-linear mean value engine model. In the studied transients the engine starts at idle and stops when the generated energy fulfills the driving mission requirement. During the driving mission both the engine speed and output power are allowed to vary, but with a constraint on power. Two strategies are then developed and evaluated. One where the driving mission is optimized with the generator power considered a free variable, and a second strategy where the accelerating phase of the transient is first optimized and then the optimal controls for a fixed generator power are used. The time optimal control is shown to be almost as fuel efficient as the fuel optimal control even though the gain in time is large. The time optimal control also has the advantage of using constant power output, making it simple and easily implementable, whilst the fuel optimal control is more complex and changes with the length of the driving mission.
\end{abstract}

\section{INTRODUCTION}

A diesel-electric powertrain, such as the BAE Systems TorqE ${ }^{\mathrm{TM}}$, see Fig. 1, has no mechanical link between the diesel engine (DE) and the wheels. This gives freedom to choose engine speed and offers a potential increase in performance as well as a potential decrease in consumption. The performance increase is due to the ability of the electric motor (EM) to provide maximum torque from standstill and the potential fuel decrease comes from that the operating point of the DE can be chosen for maximum efficiency. However

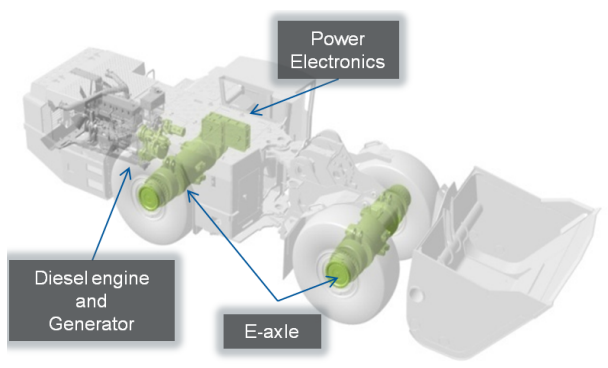

Fig. 1. BAE Systems TorqE ${ }^{\mathrm{TM}}$ powertrain.

the lack of secondary energy storage, i.e. that it is not a hybrid, provides the controller with an extra challenge since all produced energy needs to be consumed to avoid damage to the components. This paper develops and analyzes the fuel and time optimal control of a diesel electric powertrain during transients. Previous studies of optimal transient DE control have investigated the minimization of emissions during load transients for known engine speeds, see [1], [2], or studied the optimal engine operating point trajectory for a known engine power output trajectory, [3], [4]. There the DE is modeled as an inertia with a Willans-line efficiency model and the optimal solution is found using dynamic programming and Pontryagins maximum principle. Here a more detailed and complex non-linear model is used and such methods aren't feasible. Instead the problem is solved using the ACADO Toolkit, a sequential quadratic programming algorithm using multiple shooting, see [5].

This paper builds on the results in [6] and [7] where the time and fuel optimal control from idle to a target power is studied. The contribution of this paper is the study of the optimal control from idle to a target energy for two different criteria with the engine output power and engine speed considered free variables during the transient. Two different control strategies are evaluated for both criteria, one where the generater power is fixed, and one where it is considered a free variable. A nonlinear, four state, three input mean value engine model (MVEM) is used in the study. This MVEM incorporates the important turbocharger dynamics as well as the nonlinear multiple input-multiple output nature of the diesel engine. The model is also continuous and differentiable in the studied interval to enable efficient numerical solution, with the nonlinear program solver of the ACADO Toolkit.

\section{Model}

In order to study the optimal control of the DE the generator is assumed ideal, i.e. the generator efficiency is constant and the maximum power of the generator is constant over the entire speed range. The generator time constant is also assumed to be much faster than the time constant of the engine. The modeled engine is a 6-cylinder 12.7-liter SCANIA with a fixed-geometry turbine and no exhaust gas recirculation. The model used is a simplified implementation of the well validated model found in [8]. The states of the MVEM are engine speed, $\omega_{i c e}$, intake manifold pressure, $p_{i m}$, exhaust manifold pressure, $p_{e m}$, turbocharger speed, $\omega_{t c}$, and generated energy, $E_{g e n}$ and the controls are fuel flow, $u_{f}$, wastegate position, $u_{w g}$, and generator power, $P_{g e n}$. The MVEM consists of two control volumes, intake and exhaust manifold, and four restrictions, compressor, engine, turbine, and wastegate. The control 


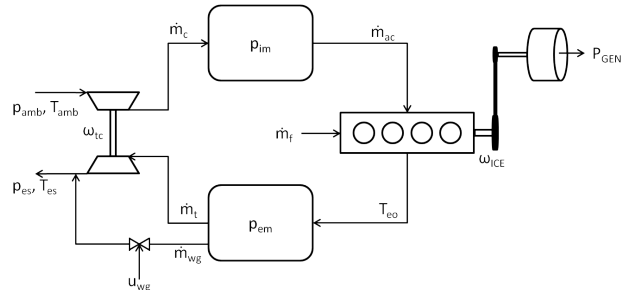

Fig. 2. Structure of the MVEM. The modeled components as well as the connection between them.

volumes are modeled with the standard isothermal model, using the ideal gas law and mass conservation. The engine and turbocharger speeds are modeled using Newton's second law. The governing differential equations of the MVEM are:

$$
\begin{aligned}
\frac{d \omega_{i c e}}{d t} & =\frac{1}{J_{g e n s e t}}\left(T_{i c e}-\frac{P_{g e n}}{\omega_{i c e}}\right) \\
\frac{d p_{i m}}{d t} & =\frac{R_{a} T_{i m}}{V_{i s}}\left(\dot{m}_{c}-\dot{m}_{a c}\right) \\
\frac{d p_{e m}}{d t} & =\frac{R_{e} T_{e m}}{V_{e m}}\left(\dot{m}_{a c}+\dot{m}_{f}-\dot{m}_{t}-\dot{m}_{w g}\right) \\
\frac{d \omega_{t c}}{d t} & =\frac{P_{t}-P_{c}}{\omega_{t c} J_{t c}}-w_{f r i c} \omega_{t c}^{2}
\end{aligned}
$$

For more details on the submodels used as well as the parameters and constants, see [6]. For more in-depth information on diesel engine modeling see [8], [9].

\section{PROBLEM Formulation}

Two non-linear optimal control problems, minimum time and minimum energy are studied. They are formulated as follows:

$$
\begin{array}{ll}
\min & \int_{0}^{T} \dot{m}_{f} \mathrm{dt} \quad \text { or } \min T \\
\text { s.t. } & \dot{x}=f(x, u),
\end{array}
$$

where $x$ is the state vector of the MVEM and $\dot{x}$ is defined by (1)-(4). In the basic transient considered in this paper the engine starts at idle when the operator requests $P_{r e q}=170 \mathrm{~kW}$. This then has to be met by the powertrain subject to constraints imposed by the components, such as maximum torque and minimum speed, as well as environmental constraints, i.e. a limit on the relationship between air and fuel flow, $\phi_{\lambda}$, set by the smoke-limiter. The constraints are:

$$
\begin{array}{ll}
x(0)=\text { idle }, & \dot{x}(T)=0 \\
T_{i c e} \leq T_{i c e, \text { max }}\left(\omega_{i c e}\right), & P_{\text {gen }}(T)=P_{\text {req }} \\
\omega_{i c e} \geq \omega_{\text {ice, } \text { min }}, & \phi_{\lambda} \geq 0
\end{array}
$$

In a vehicle equipped with a diesel-electric powertrain the demand from the operator can be interpreted as a electric power request, the interpretation of (5)-(6) is thus how to meet this power request either as fast or as fuel efficient as possible.

\section{Optimal Power Transients}

The optimal torque and speed trajectories to problem (5)(6) are shown in Fig. 3. It can be seen that the time and fuel optimal power transients are quite similar in structure,

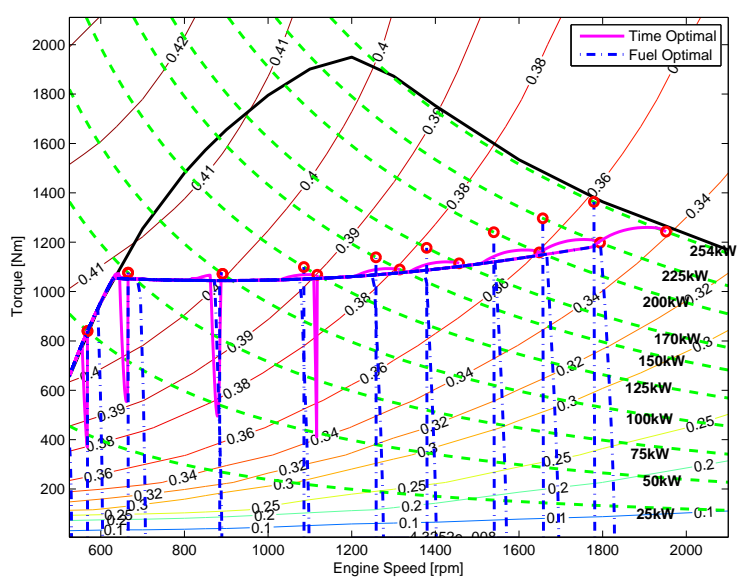

Fig. 3. Time and fuel optimal solutions to different load transients. The time and fuel optimal transients have similar structures, dictated by the smokelimiter and maximum torque limit, but differ in how they meet the end constraints.

but the end-points differ. This structure is mainly to input as much energy into the system as possible, i.e. follow the maximum torque line and the $\lambda_{\min }$ set by the torque limiter. The difference between the two criteria is which end-point is approached and how it is approached. The fuel optimal transients approach a stationary point with higher turbocharger speed than the time optimal transients. For a more extensive discussion on power transients see, [6] and [7].

\section{A. Transients between two output powers}

The transients shown in Fig. 3 are all from idle to target power. The initial point however does not change the characteristics of the solution, as seen in Fig. 4. Since there is a difference in initial conditions the solution approaches slightly different end operating points, the manner in which they do it is however the same.

\section{B. Transients to the fuel optimal operating point}

As seen in Fig. 3 none of the fuel optimal transients end in the fuel optimal operating point for that power. To reach the more efficient region of the engine map, more kinetic energy has to be stored in the turbocharger without increasing the kinetic energy in the engine. To accomplish this the engine needs to be braked by generator, that is, energy has to be removed from the system. This is clearly not optimal if the aim is just to go from idle to a target power. However it does raise the question of how to, in a fuel optimal way, go to the fuel optimal operating point, and also how much it costs. In Fig. 5 fuel optimal transients are compared to fuel optimal transients to the fuel optimal operating point. These transient take roughly twice as long as the fuel optimal transients, and consume roughly three times the fuel. This comparison is however not entirely valid since the generated energy is also roughly 200 times larger. Whether this is optimal or not depends on what happens after the transient. 

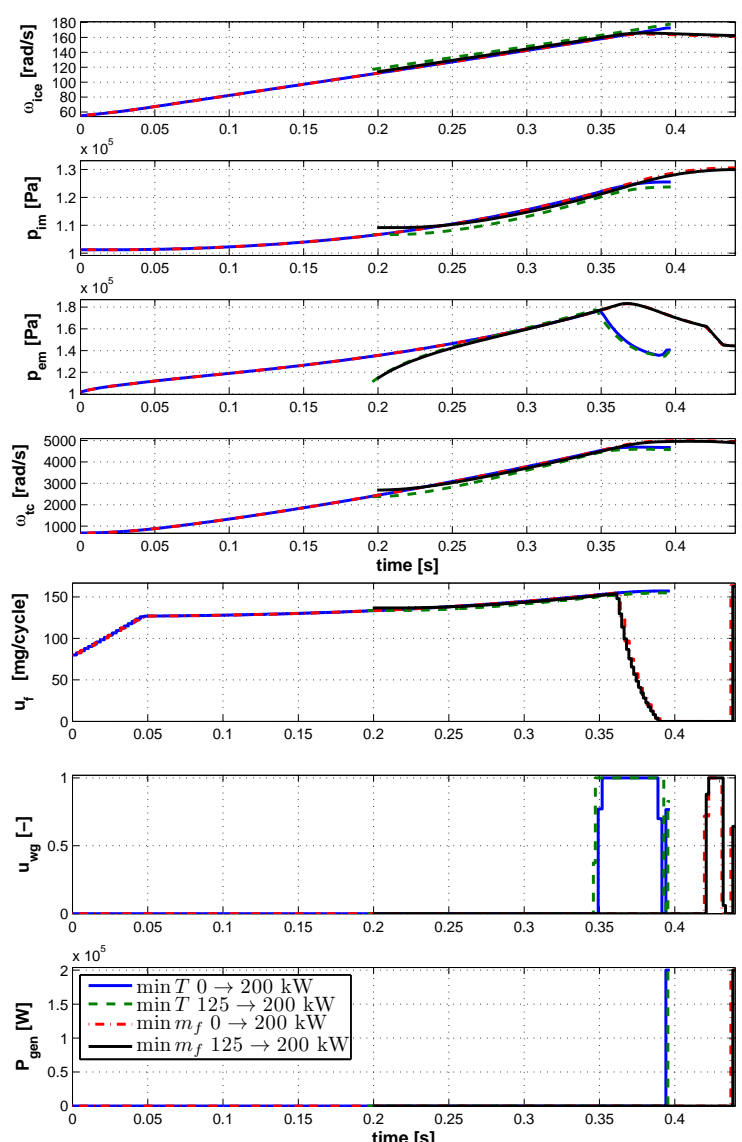

Fig. 4. Transients from idle to $200 \mathrm{~kW}$ as well as transients from $125 \mathrm{~kW}$ to $200 \mathrm{~kW}$

\section{Extending the Optimal Power Transients to DRIVING MISSIONS}

In Section IV the optimal trajectories for steps in requested power is presented. A driving mission normally doesn't end when the requested power has been met, output power is usually requested for a period of time. Instead a driving mission for off-highway vehicles is often to move something between two locations. The driving mission can thus be defined as that a certain amount of energy has to be produced. This is achieved by extending the problem defined in (5)-(6) with (7).

$$
\int_{0}^{T} P_{g e n} \mathrm{dt}=E_{r e q}
$$

A tempting way to approach this problem is to separate it into two problems. First use the results from Section IV to go in an optimal manner to the requested power. Then solve a second optimization problem starting in that point, with $P_{\text {gen }}=P_{\text {req }}$ until (7) is fulfilled. This strategy is denoted $S-P E$ and is defined as:

$$
\begin{aligned}
S-P E_{m_{f} / T}: & E_{g e n}(T)=0, P_{g e n}(T)=P_{r e q} \\
E_{g e n}(T) & =E_{r e q}, P_{g e n}=P_{r e q}
\end{aligned}
$$

The idea of $S-P E$ is mainly valid when the criterion is to minimize the fuel consumpion since in the second optimization problem $P_{g e n}$ and $E_{g e n}$, and thus $T$, are fixed.
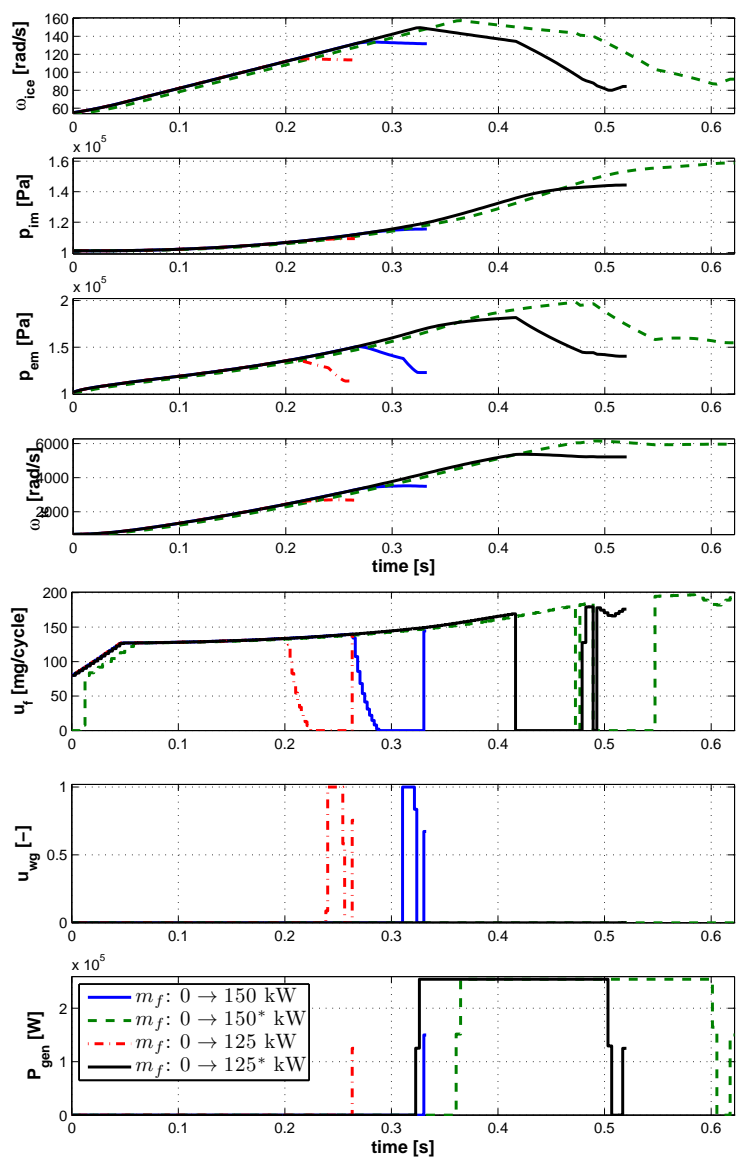

Fig. 5. Comparison between fuel optimal transients and transients to the fuel optimal operating point. $*$ denotes the fuel optimal operating point.

In the time optimal case the solution is to hold the end controls from the first optmization problem for the duration $T$. The resulting control and state trajectories for $S-P E$ for $E_{r e q}=[170680] \mathrm{kJ}$ are shown in Fig. 6. The characteristics of the solution is the same for both $1 \mathrm{~s}$ and $4 \mathrm{~s}$. For $S-P E_{m_{f}}$ the solution is to stop the fuel injection for the last parts of the first phase as well as actuate the wastegate to get stationarity, as shown in [6]. The second phase then starts with fuel cut-off and the kinetic energy of the engine being used to generate power, see Fig. $6, t \in[0.33,0.44]$. After that the solution is to approach the point of maximum efficiency for the requested power. The control then ends in an operating point that is not the optimal operating point, due to that the decrease in fuel consumption of going there is larger than the increase in fuel consumption of being there in the last time step. So the only difference that appears when the length of the mission changes is how far towards the peak efficiency for that power the solution has time to wander. For $S-P E_{T}$ the solution is just to maintain the end controls of phase 1 for the duration of the mission.

A problem with the $S-P E$ strategies is that they don't fully utilize the kinetic energy of the engine to generate power. In min $\mathrm{T}$ the engine speed is at a stationary high value and in $\min m_{f}$ the engine speed is decreased at the end of phase 1 without using that energy to generate power. But since the 

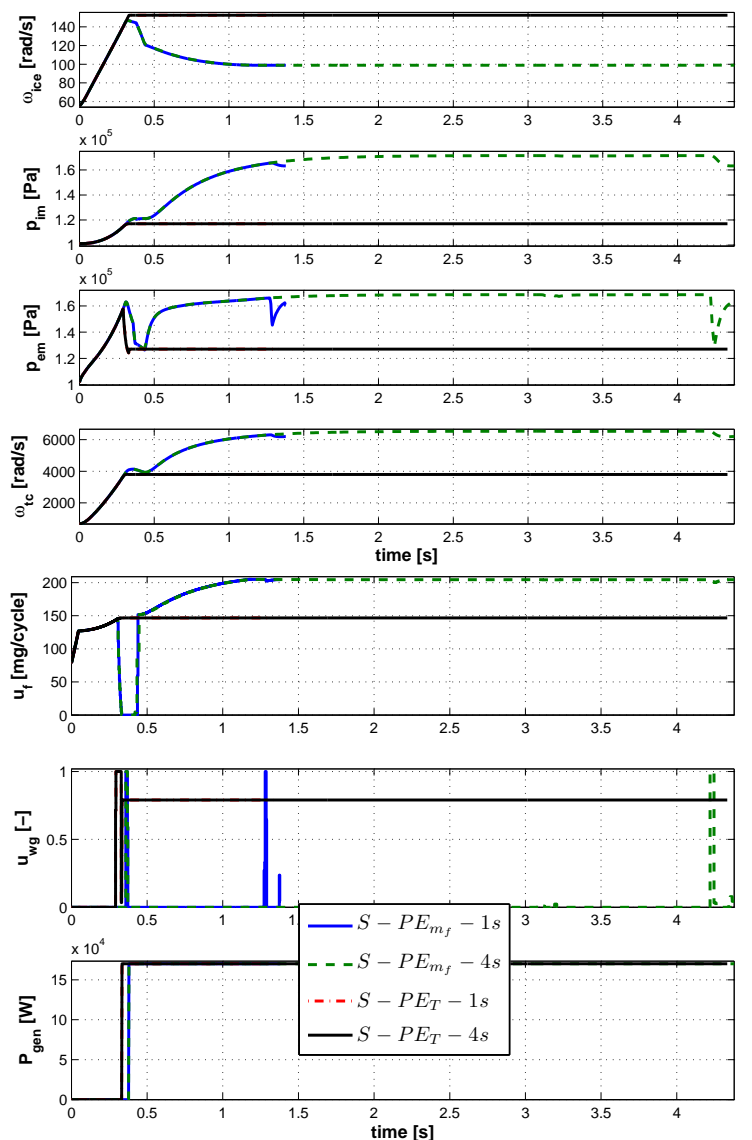

Fig. 6. State and control trajectories for $S-P E_{m_{f}}$ and $S-P E_{T}$ with $P_{r e q}=170 \mathrm{~kW}$ for $T$ equal to 1 and $4 \mathrm{~s}$. That is $E_{r e q}=\left[\begin{array}{ll}170 & 680\end{array}\right] \mathrm{kJ}$.

output power is constant the control strategy becomes rather straight forward both for time and fuel optimal transients. Use the optimal control to target power, and then either wander towards maximum efficiency, for fuel optimal control, or just use constant control, for time optimal control.

\section{Optimal EnERgy TRAnSIEnTs}

In order to gain understanding of the optimal control during a driving mission and also to evaluate how far from optimal the $S-P E$ is, a different optimization problem is solved. In this optimization problem the output power is not a constraint, instead the control is required to fulfill the driving mission, which is defined as that it has to provide a certain amount of energy. The duration and the rate at which the energy is provided is allowed to vary freely as long as it does not exceed the request of the operator. This strategy, denoted $S-E$, can be formulated as a set of constraints according to:

$S-E_{m_{f} / T}: E_{g e n}(T)=E_{r e q}, P_{g e n} \leq P_{r e q}, P_{g e n}(T)=P_{r e q}$

This can be interpreted as how large portions of the transients are to be optimized before the control has to fulfill the drivers request to produce a certain power.

\section{A. Time Optimal Energy Transients}

The time optimal control and state trajectories for $P_{r e q}=$ $170 \mathrm{~kW}$ and $E_{\text {req }}=\left[\begin{array}{llll}170 & 340 & 510 & 680\end{array}\right] \mathrm{kJ}$ are shown in
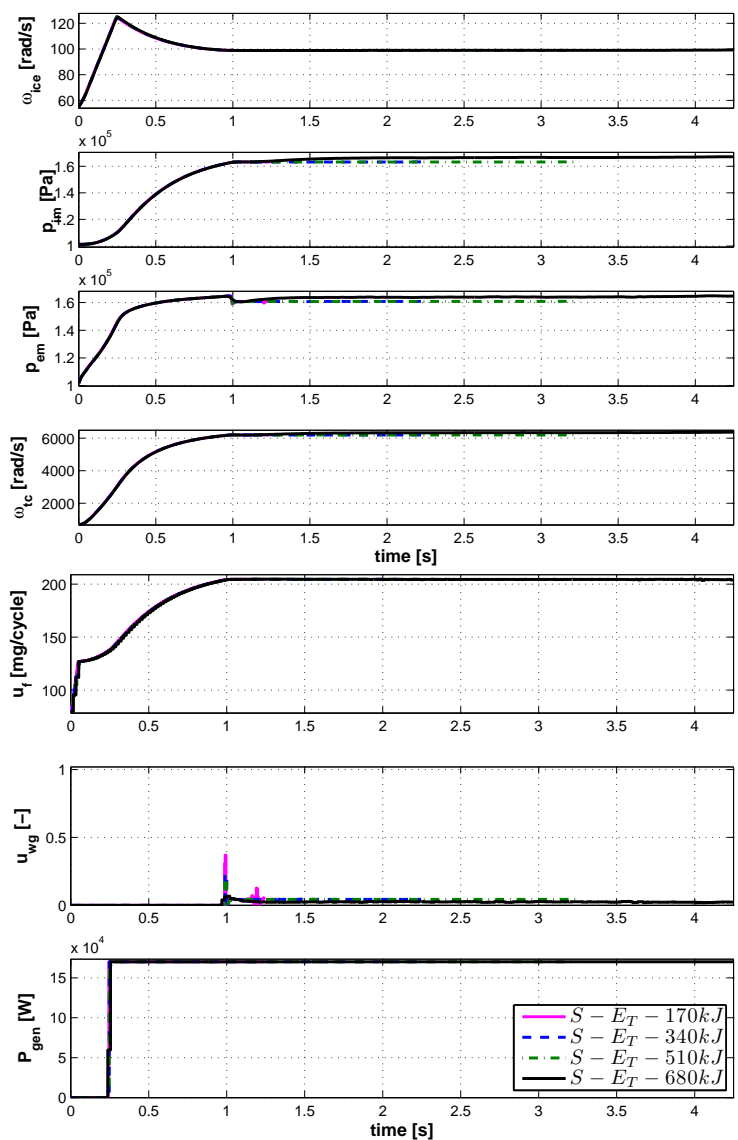

Fig. 7. State and control trajectories for $S-E_{T}$ for $E_{r e q}=$ [170 340510680$] \mathrm{kJ}$

Fig. 7. Regardless of the amount of required energy the characteristics of the solution doesn't change. The optimal solution is to first accelerate the engine with maximum torque to $\omega_{i c e} \approx 120 \mathrm{rad} / \mathrm{s}$ and then start to produce power while at the same time converting the kinetic energy. The solution is rather stationary after roughly $1 \mathrm{~s}$. This in combination with the amount of requested energy and the constraint that the requested power can not be exceeded results in all solutions being similar regardless of $E_{r e q}$.

\section{B. Fuel Optimal Energy Transients}

The fuel optimal control and state trajectories for $P_{r e q}=$ $170 \mathrm{~kW}$ and $E_{r e q}=\left[\begin{array}{llll}170 & 340 & 510 & 680\end{array}\right] \mathrm{kJ}$ are shown in Fig. 8. The characteristics of the solution changes as $E_{r e q}$ changes. For $E_{r e q}=\left[\begin{array}{ll}170 & 340\end{array}\right] \mathrm{kJ}$ the optimal control follow the same characteristic. The first phase of the control is to start generating power early and then follow the maximum torque line. Then in order to fulfill the end power requirement the engine departs from the maximum torque line as it accelerates and approaches the stationary value from a higher engine speed following the same line as $S-P E_{m f}$ and $S-E_{T}$, see Fig. 9.

For $E_{r e q}=\left[\begin{array}{ll}510 & 680\end{array} \mathrm{~kJ}\right.$ the engine is accelerated before energy is produced as can be seen in Fig. 9 the controls follows the same trajectory, dictated by the smoke-limiter 
and maximum torque line, as $S-P E_{T / m_{f}}$, that is, as the power transients presented in Section IV. How far along this trajectory they wander differs between the two. For $E_{r e q}=510 \mathrm{~kJ}$ the over-shoot in engine speed is very small before it starts to approach the maximum torque line which it then follows to the end point. For $E_{r e q}=680 \mathrm{~kJ}$ the engine is accelerated to a higher engine speed, almost in the manner of $S-E_{T}$, before the generator starts to produce power. The controls also approach something that can be considered a stationary point before the end point is approached. The end point for $E_{r e q}=[510680] \mathrm{kJ}$ is also approached following the maximum torque line. For low requested energies the control is to follow the maximum torque line and then accelerate the engine, for higher requested energies this is done in the reverse order, first accelerate the engine and then follow the maximum torque line.

Interesting to note is that none of the $S-E_{m_{f}}$ approach the operating point with maximum efficiency. For $E_{r e q}=$ [170 340 510] $\mathrm{kJ}$ the driving missions are so short that they do not approach any stationary point, the whole mission is a transient in order to fulfill the end constraints. However $E_{\text {req }}=680 \mathrm{~kJ}$ approaches a stationary point, but not the point of peak efficiency, but instead the maximum efficiency for $P_{g e n}=P_{r e q}$. The explanation for this is believed to be that the driving mission is still too short. If it would approach the point of peak efficiency the mission would end with another transient in order to fulfill the end constraints and since the driving mission is short the benefits of being at the point of maximum efficiency would be lost in the second transient.

\section{RESUlTS AND DisCUSSION}

To be able to compare the different strategies and requested energies all controls are augmented so that they all produce $680 \mathrm{~kJ}$, i.e. all shorter driving missions are extended, by maintaining the end point until the target energy is reached. Note that $S-P E_{T}$ are all the same after augmentation since the second phase just holds the final controls of the first phase. The results are shown in Fig. 9 and Table I, where the augmented $S-E_{m_{f}}, S-E_{T}, S-P E_{m_{f}}$ are all compared relative $S-P E_{T}$. Since the trajectories of $S-E_{T}$ and $S-P E_{m_{f}}$ do not change with $E_{r e q}$ only $E_{r e q}=680 \mathrm{~kJ}$ is shown. In Table I it can be seen that the difference between different requested energies is small, in the fourth and fifth digit. These small variations are influenced by the tolerances and discretizations set for the solver, conclusions can therefore not be drawn from the small variations that are found within a problem formulation. The focus in the comparison is on the difference between the time and fuel optimal formulations, where clear conclusions can be drawn. Different initial guesses have been used to try and ensure that the attained solutions are not local minima. The problems solved here are on the limit, regarding size and complexity, of what currently can be solved with the software.

For $S-P E_{m_{f}}$ the increase in time compared to $S-P E_{T}$ is around $1 \%$ but the decrease in fuel consumption is roughly $11 \%$. Since the time is only optimized in the first phase,
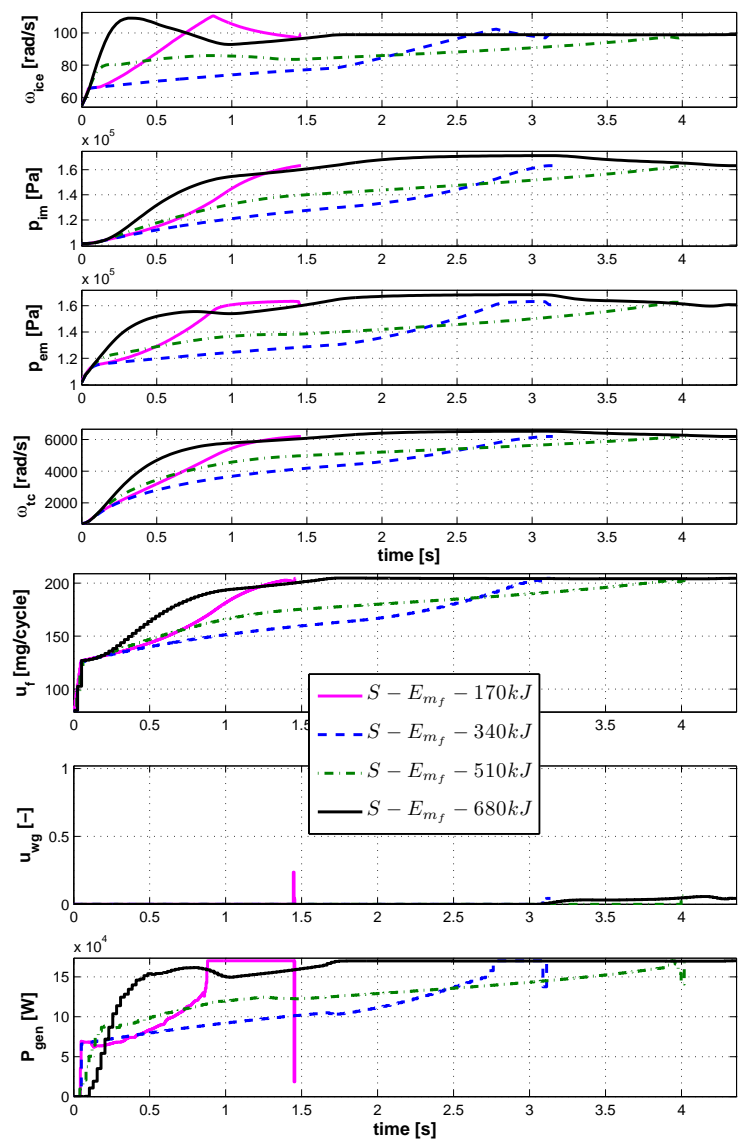

Fig. 8. State and control trajectories for $S-E_{m_{f}}$ for $E_{r e q}=$ [170 340510680$] \mathrm{kJ}$

all solutions have the same length after augmentation. That increasing $E_{r e q}$ has a small effect on the fuel consumption is probably due to that the solution of the second optimization problem departs from the optimal operating point at the end of the transient since the decrease of shifting operating point is larger than the increase of being away from the optimal operating point in the last sample. Therefore the control augmented the least has the lowest consumption, the difference is however negligible. Despite the simplicity of this strategy it performs relatively well compared to the other strategies.

Interesting to note is that the trajectories of $S-E_{T}$ are quite similar to $S-P E_{m_{f}}$, even though the criterion is different. The $S-E_{T}$ is however both faster and consumes less fuel. As previously discussed the $S-P E_{m_{f}}$ doesn't make use of all the stored kinetic energy and this is probably the main reason for this difference. The decrease in time and fuel consumption is $2 \%$ and $12 \%$ respectively. The slight increase in time consumption with $E_{r e q}$ is probably due to different control interval to time ratios used.

As seen in Fig. 8 and Fig. 9 the control for $S-E_{m_{f}}$ changes with $E_{r e q}$. For all $E_{r e q}$ the fuel consumption reduction is around $12 \%$, the time consumption however increases with $1-19 \%$. Interesting to not is that the fuel consumption increase from minimizing time is small, only up to $0.4 \%$, but not only is the time consumption lower, the control is more straight 
forward. In $S-E_{m_{f}}$ the produced power varies, something that might not be desirable, which is avoided with $S-E_{T}$.

\section{CONCLUSIONS}

Optimal control of a diesel-electric powertrain during transients is studied. First the minimum fuel and minimum time control to steps in requested powers is discussed. The power transients are then extended to shorter driving missions. The driving missions are defined as that the powertrain has to produce a certain amount of energy. This can be interpreted as how much freedom the optimization has when the operator applies a step in requested power before this power has to be produced.

Two different control strategies for these driving missions as well as how they change with the amount of requested energy are studied, both for minimum time and minimum fuel. The first strategy, denoted $S-P E$, solves two optimal control problems. First the optimal control for a step in power request is applied, then the control with constant output power is optimized. The second strategy, denoted $S-E$, solves one optimal control problem where the generator power is considered a free variable.

For $S-P E_{T}, S-P E_{m_{f}}$ and $S-E_{T}$ the characteristics of the solution does not change with requested energy. The optimal solution is to accelerate the engine, following the smoke-limiter, and then use the excess kinetic energy to produce power and approach the maximum efficiency point for the requested $\operatorname{power}\left(S-P E_{m_{f}}\right.$ and $\left.S-E_{T}\right)$, or to just stay in the end point achieved when just the power transient is optimized $\left(S-P E_{T}\right)$. The $S-E_{m_{f}}$ changes with $E_{r e q}$. For lower requested energy the solution is to follow the maximum torque line, then accelerate the engine whilst producing power, and then finally approach the end operating point from a higher engine speed. For higher requested energies the engine is accelerated before power is produced, the end point is then approached following the maximum torque line.

The strategies are evaluated relative $S-E_{T}-680 \mathrm{~kJ}$. In order for this to be possible the controls for lower requested energies are augmented by holding on to the final operating point until the requested energy is produced. The result shows that compared to the $S-P E_{T}$ large gains in fuel economy can be made. However, all evaluated controls offer roughly a $12 \%$ reduction in consumption, but the time required differs. Interesting to note is that $S-E_{T}$ has lower fuel consumption than $S-P E_{m_{f}}$ and almost as low as $S-E_{m_{f}}$ even though it is time that is minimized. This strategy thus offers almost the optimum fuel economy but is $2-20 \%$ faster, depending on amount of requested energy. None of the controls approach the peak efficiency point. This is believed to be due to the short nature of the driving missions in combination with the end constraints.

\section{ACKNOWLEDGMENT}

The support from BAE Systems in Örnsköldsvik and the funding from the Swedish Energy Agency FFI is gratefully acknowledged.

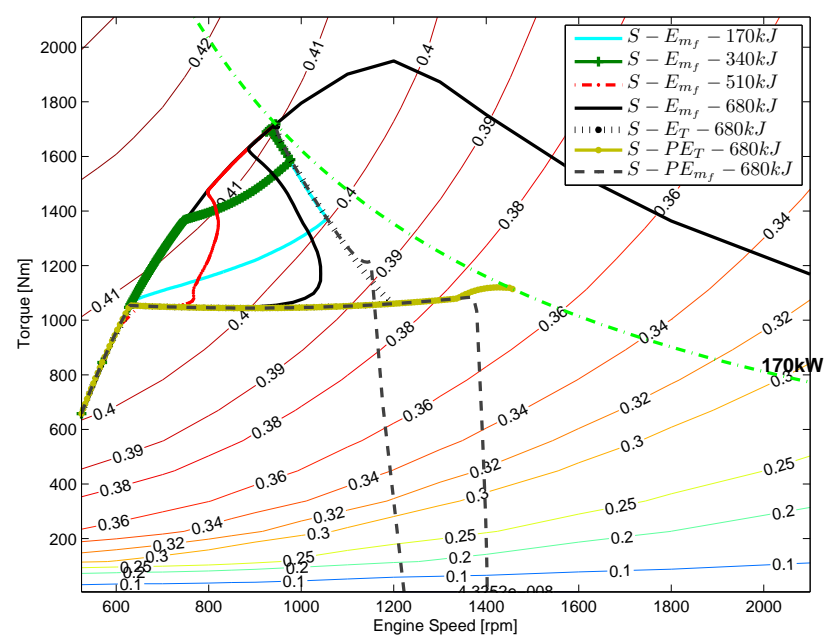

Fig. 9. Torque vs engine speed for $S-E_{T / m_{f}}$ and $S-P E_{m_{f}}$ compared to $S-P E_{T}$ for different $E_{r e q}$.

TABLE I

THE CHANGE IN FUEL CONSUMPTION AND DURATION OF THE DIFFERENT STRATEGIES COMPARED TO $S-P E_{T}-680 \mathrm{KJ}$.

\begin{tabular}{ccccc}
\hline \hline$E_{r e q}(T)$ & $\min$ & $S-P E_{m_{f}}$ & $S-E_{m_{f}}$ & $S-E_{T}$ \\
\hline \multirow{2}{*}{ 170kJ } & $\Delta T[\%]$ & 1.07 & 2.92 & -2.08 \\
& $\Delta m_{f}[\%]$ & -11.44 & -11.95 & -11.85 \\
\hline \multirow{2}{*}{$340 \mathrm{~kJ}$} & $\Delta T[\%]$ & 1.07 & 18.56 & -2.01 \\
& $\Delta m_{f}[\%]$ & -11.45 & -12.14 & -11.84 \\
\hline \multirow{2}{*}{$510 \mathrm{~kJ}$} & $\Delta T[\%]$ & 1.07 & 16.26 & -1.99 \\
& $\Delta m_{f}[\%]$ & -11.45 & -12.25 & -11.83 \\
\hline \multirow{2}{*}{ 680kJ } & $\Delta T[\%]$ & 1.07 & 0.66 & -1.96 \\
& $\Delta m_{f}[\%]$ & -11.46 & -11.95 & -11.81 \\
\hline
\end{tabular}

\section{REFERENCES}

[1] M. Benz, M. Hehn, C. H. Onder, and L. Guzzella, "Model-based actuator trajectories optimization for a diesel engine using a direct method," Journal of Engineering for Gas Turbines and Power, vol. 133, 2011.

[2] N. P. Kyrtatos, E. I. Tzanos, and C. I. Papadopoulos, "Diesel engine control optmization for transient operation with lean/rich switches," Journal of Engineering for Gas Turbines and Power, vol. 4, no. 3, pp. 219-232, 2003.

[3] T. Nilsson, A. Fröberg, and J. Åslund, "Optimized engine transients," in 7th IEEE Vehicle power and propulsion conference, Chicago, Illinois, USA, 2011.

[4] - "Optimal operation of a turbocharged diesel engine during transients," in SAE Technical Paper 2012-01-0711, 2012.

[5] B. Houska, H. J. Ferreau, and M. Diehl, "ACADO toolkit - an open source framework for automatic control and dynamic optimization," Optimal Control Applications \& Methods, vol. 32, no. 3, pp. 298-312, 2011.

[6] M. Sivertsson and L. Eriksson, "Optimal power response of a dieselelectric powertrain," in IFAC Workshop on Engine and Powertrain Control, Simulation and Modeling, Paris, France, 2012.

[7] — , "Optimal step responses in diesel-electric systems," in Submitted to the 13th Mechatronics Forum International Conference, Linz, Austria, 2012.

[8] J. Wahlström and L. Eriksson, "Modelling diesel engines with a variablegeometry turbocharger and exhaust gas recirculation by optimization of model parameters for capturing non-linear system dynamics," Proceedings of the Institution of Mechanical Engineers, Part D, Journal of Automobile Engineering, vol. 225, no. 7, pp. 960-986, 2011.

[9] L. Eriksson, "Modeling and control of turbocharged SI and DI engines," Oil \& Gas Science and Technology - Rev. IFP, vol. 62, no. 4, pp. 523-538, 2007. 